\title{
Study and analysis of an electric Z-shaped meta-atom
}

\author{
Abdallah Dhouibi ${ }^{1}$, Shah Nawaz Burokur ${ }^{2,3^{*}}$, André de Lustrac ${ }^{2,3}$, and Alain Priou ${ }^{1}$ \\ ${ }^{1}$ LEME, Univ. Paris-Ouest, EA 4416, 92410 Ville d'Avray, France \\ ${ }^{2}$ IEF, Univ. Paris-Sud, CNRS, UMR 8622, 91405 Orsay cedex, France \\ ${ }^{3}$ Univ. Paris-Ouest, 92410 Ville d'Avray, France \\ *corresponding author, E-mail: shah-nawaz.burokur@u-psud.fr
}

\begin{abstract}
A printed Z-shaped electric meta-atom is presented as an alternative design to the conventional electric-LC (ELC) resonator. We propose to redesign the ELC resonator pattern to get a compact and a low cost electric resonator exhibiting a strong electric response. Our approach consists in redressing the resonator shape to accommodate higher inductance and therefore leading to a lower resonance frequency without being limited by fabrication tolerances. Simulation and measurement results show that the $\mathrm{Z}$ metaatom exhibits an electric response to normally incident radiation and can be used very effectively in producing materials with negative permittivity.
\end{abstract}

\section{Introduction}

Metamaterials offer remarkable properties that go beyond conventional natural materials and are actually used in novel class electromagnetic devices such as invisibility cloaks [1-5], rotators [6], retroreflectors [7], Luneburg lenses [8], waveguide tapers [9] and directive antennas [1017]. A typical metamaterial is an artificially engineered structure made of a periodic array of sub wavelength metallic or dielectric inclusions called meta-atoms. Various forms of meta-atoms such as Split Ring Resonator (SRR) [18] or electric-LC (ELC) resonator [19] have been shown to be able to produce respectively a wide range of permeability or permittivity values in the vicinity of the resonance. Other metamaterial resonators such as the Sshaped structures $[20,21]$ and the asymmetric cut wire pairs [22-24] have shown a negative refractive index. In order to use such resonators in the effective medium regime, the ratio between the operating wavelength and the unit cell size must be greater than 4 [25]. Different approaches have been proposed to increase the latter ratio with the main goal to shift downwards the resonance frequency of the metaatoms. For instance, solutions such as integrating interdigital capacitors [26] or using high permittivity or permeability host substrates [27] have been proposed. However, such attempts are very limited according to the fabrication technology when frequency shifts upwards.

It is our aim in this paper to investigate numerically and experimentally the electromagnetic properties of a Z-shaped meta-atom in the microwave regime. The latter meta-atom is obtained by changing the LC equivalent circuit topology of the ELC resonator. It is reported that the structure presents an electric resonance which occurs at a lower frequency than that presented by the ELC resonator for similar geometrical dimensions. A negative real part of $\varepsilon$ is demonstrated in the vicinity of the resonance. Moreover, the experimental study of cross-polarization and magnetoelectric coupling in such an electric Z-shaped resonator is presented. The results are further compared with those of completely symmetric ELC and electric I-shaped resonators. It is shown that cross-polarization is near zero in the resonance vicinity of the $Z$-shaped resonator, indicating that a purely electric response is obtained at resonance.

\section{From ELC to Z-shaped resonator}

We first consider the unit cell of the ELC resonator introduced in Ref. 19 and presented in Fig. 1(a). In the effective medium regime, where resonator size is much smaller than the operating wavelength, the ELC resonator can be described qualitatively in terms of its equivalent circuit as illustrated in Fig. 1(b). A capacitor-like structure couples to the electric field and is connected in parallel to two loops providing inductance to the circuit. In order to increase the ratio between the operating wavelength and the ELC unit cell size it has been suggested that the resonance frequency of the LC circuit expressed as $f_{r}=\frac{1}{2 \pi \sqrt{L C}}$, can be tuned downwards by introducing additional inductive loops to accommodate more inductance. Similar to interdigital capacitors, adding inductive loops can be a difficult technological task at optical frequencies.

Thus, we propose to transform the LC topology of the ELC resonator with the main goal to lower the resonance frequency. This transformation presents also the advantage of facilitating the metamaterial fabrication process. Hence, the ELC resonator can be easily reduced to an I-shaped resonator by removing firstly the two side arms, causing the inductance of the element to considerably decrease. On the counterpart, a new capacitance is created between the two horizontal arms. However, this capacitance $\mathrm{C}$ is very weak compared to that of the gap in the middle of the structure, which suggests that the transformed resonator will show a higher resonant frequency than the ELC. Removing the gap 
in the middle of the resonator changes the LC circuit topology and the resulting I-shaped resonator presented in Fig. 1(c) is equivalent to the circuit given in Fig. 1(d). In this case without the gap, the capacitance needed for the LC resonance is given by $C_{p}$ created between two consecutive unit cells

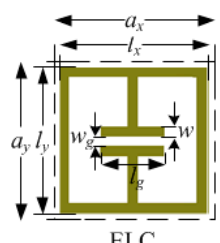

(a)

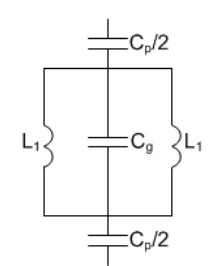

(b)

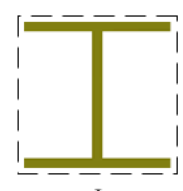

(c)

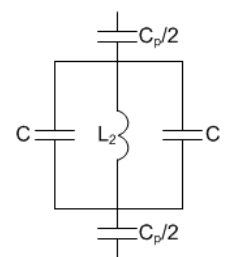

(d)

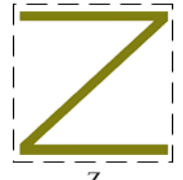

(e)

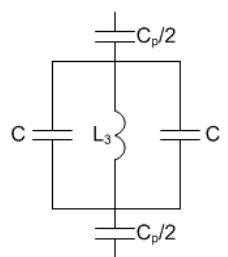

(f)

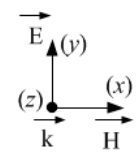

Figure 1: Unit cells and equivalent LC circuit models. (a)(b) ELC resonator. (c)-(d) I-shaped resonator. (e)-(f) Zshaped resonator. For all the resonators, the geometrical dimensions are: $a_{x}=a_{y}=6 \mathrm{~mm}, l_{x}=5.7 \mathrm{~mm}, l_{y}=5.8 \mathrm{~mm}, w$ $=0.3 \mathrm{~mm}, w_{g}=0.45 \mathrm{~mm}$ and $l_{g}=2 \mathrm{~mm}$.

Another practical shape transformation aiming to decrease the resonance frequency of the ELC resonator without changing the lattice constant consists in adding physical length to both inductive loops by using a slant line as presented in Fig. 1(e). Adding physical length when passing from I-shaped to Z-shaped structure shall produce a larger phase delay and increases the total inductance of the metamaterial without requiring additional fabrication steps. Due to this increase in inductance, the Z-shaped resonator shall present resonance at a lower frequency. The equivalent circuit model of the resulting Z-shaped structure is given in Fig. 1(f).

\section{Parametric analysis of the resonators}

To examine and compare the performances of the different resonators presented in Fig. 1, the properties of the structures are characterized numerically using a Finite Difference Time Domain (FDTD) Maxwell's equations solver, Microwave Studio Suite by CST. The dielectric spacer used throughout this study is single face coppercladded epoxy with a relative dielectric constant of 3.9, a tangential loss of 0.02 and a thickness $t_{s}=0.4 \mathrm{~mm}$. For the samples reported in this paper, the nominal length of the 17 $\mu \mathrm{m}$ thick copper wires along the $\mathrm{x}$ - and $\mathrm{y}$-direction is respectively $l_{x}=5.7 \mathrm{~mm}$ and $l_{y}=5.8 \mathrm{~mm}$ and the nominal width is $w=0.3 \mathrm{~mm}$ in both simulations and experiments. For the capacitive gaps present in the ELC, the nominal width and length is respectively $w_{g}=0.45 \mathrm{~mm}$ and $l_{g}=2$ $\mathrm{mm}$. For the unit cell, $a_{x}=a_{y}=6 \mathrm{~mm}$. For an electromagnetic wave incident with a wave vector and field polarization of Fig. 1, the different resonators will exhibit a strong electric response.

A parametric study of the resonators is performed in order to point out the importance of the different geometric parameters in the LC resonance frequency and to provide a guideline for design of such metamaterials. Three series of calculations are performed for each resonator; the first one concerns the width of the strip lines $w$, the second one for the length $l_{y}$ and the third one concerns the length $l_{x}$. The transmission spectra under normal incidence are shown for each configuration. The parametric study is presented in the various parts of Fig. 2. Each column corresponds to a specific resonator and each row corresponds to the study of a physical parameter. In all subplots, the green traces correspond to the nominal values and the red and blue traces correspond respectively to upper and lower values. When considering only the resonance corresponding to nominal parameters (green traces), a resonance is clearly observed for each type of meta-atom. The I-shaped resonator presents a resonance frequency of $6.4 \mathrm{GHz}$, compared to $5.9 \mathrm{GHz}$ for ELC. This shift toward higher frequencies was predictable since the inductance and capacitance are reduced when transforming ELC into Ishaped resonator. The shape transformation induces a change on the LC topology. In a quasi-static approximation, where resonator size is very small compared to operating wavelength, the I-shaped structure can be modeled in the form of the LC resonance circuit presented in Fig. 1(d). The equivalent capacitance in the I-shaped structure is then given by $C_{p}$ since we neglect the capacitance $C$. According to the dimensions $w_{g}$ and $l_{g}$ used for the gap of the ELC, the capacitance of the latter $\left(C_{g}\right)$ is almost half of the one created by the periodicity $\left(C_{p}\right)$.

The resonance frequency of the $\mathrm{Z}$-shaped resonator is expressed as $f_{r}=\frac{1}{2 \pi \sqrt{L_{3} C_{p}}}$ where the inductance is approximated by $L_{3} \approx \mu_{0} t_{s}\left(\frac{2 w}{l_{x}}+\frac{\sqrt{l_{x}^{2}+l_{y}^{2}}}{w}\right)$ and the inter-cell capacitance by $C_{p} \approx \frac{\varepsilon l_{x}}{\pi} \ln \left(\frac{2 w}{a_{y}-l_{y}}\right) \quad[18]$. The circuit theory then predicts a resonance frequency equal to $5.2 \mathrm{GHz}$, which is in good agreement with the simulated value. Concerning the I-shaped resonator, the inductance is approximated by $L_{2} \approx \mu_{0} t_{s}\left(\frac{2 w}{l_{x}}+\frac{l_{y}}{w}\right)$ and the calculated resonance frequency is $6.2 \mathrm{GHz}$. The equivalent capacitance of the ELC resonator is given by $\frac{C_{p} C_{g}}{C_{p}+C_{g}} \approx \frac{C_{p}}{3}$, and it's inductance is given by 
ELC
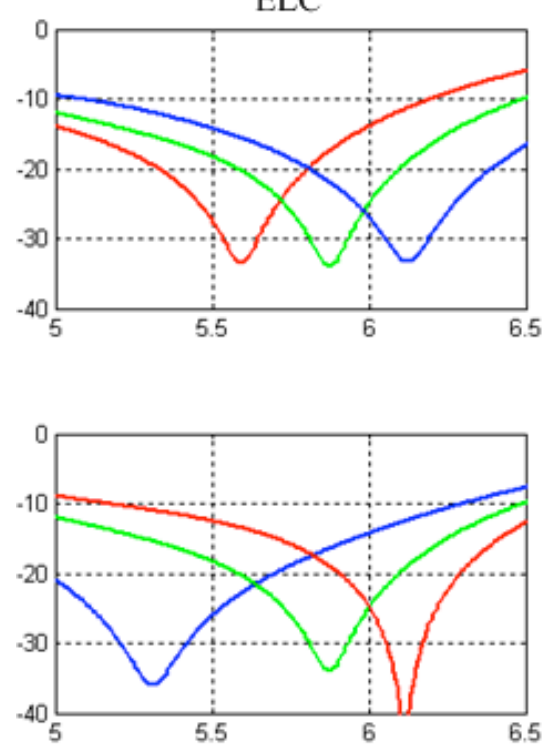

I
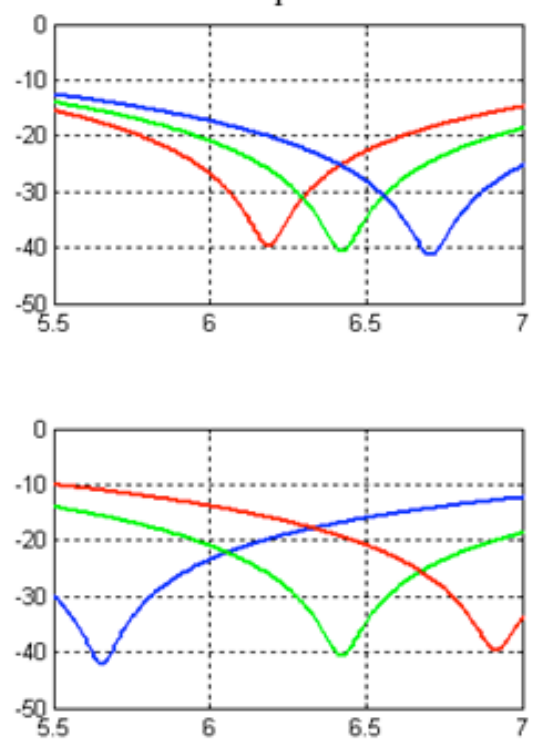

Z

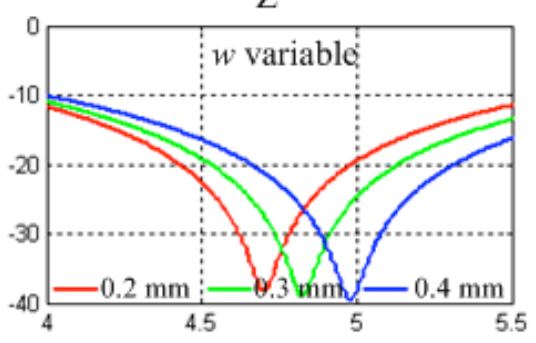

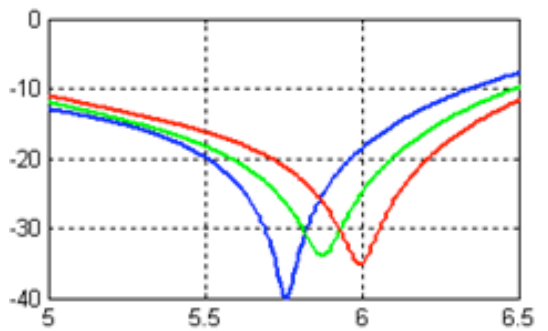
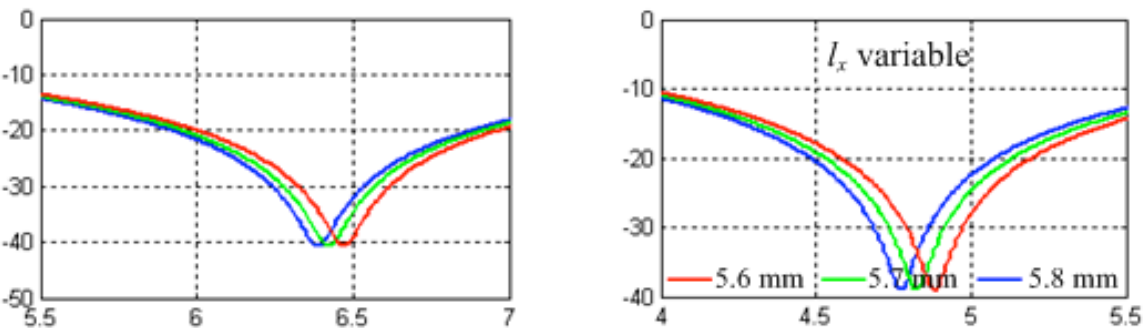

Figure 2: Parametric study of ELC, I-shaped and Z-shaped resonators. Variation of $w, l_{y}$ and $l_{x}$.

$L_{1} \approx \mu_{0} t_{s}\left(\frac{2 w}{l_{x}}+\frac{2 l_{y}}{w}+\frac{l_{y}-2 w-w_{g}}{w}\right)$. Hence, circuit

theory predicts a resonance frequency of $6.1 \mathrm{GHz}$ for the ELC resonator. A good qualitative agreement can be observed with the resonance frequencies obtained from CST simulations as shown in Table I. The effective medium ratio $\lambda / \mathrm{a}$, i.e. the ratio between the operating wavelength and the unit cell size, is also given for each meta-atom in Table I. We can clearly observe that parameters extraction is indeed possible with the different resonators since $\lambda / a$ is greater than 4 .

Table 1: Computed and measured resonance frequencies of the resonators $(\mathrm{GHz})$.

\begin{tabular}{ccccc}
\hline Resonator & $\begin{array}{c}\text { Simulated } \\
f_{0}\end{array}$ & $\begin{array}{c}\text { Equivalent } \\
\text { circuit } f_{0}\end{array}$ & $\begin{array}{c}\text { Measured } \\
f_{0}\end{array}$ & $\begin{array}{c}\text { Simulated } \\
\lambda / \mathrm{a}\end{array}$ \\
\hline ELC & 5.9 & 6.1 & 6 & 8.5 \\
I & 6.4 & 5.9 & 6.5 & 7.8 \\
Z & 4.9 & 5.1 & 4.9 & 10.2 \\
\hline
\end{tabular}

Commenting on the parametric sweep, the first series of calculation given in Fig. 2 illustrate the influence of the strip lines width, $w$. We observe a shift toward higher frequencies for the three resonators when increasing $w$, since the inductance involved in the LC resonance is reduced. The second series of calculation is based on the variation of the vertical length $l_{y}$, and keeping $a_{y}$ fixed to 6 $\mathrm{mm}$. As $l_{y}$ is increased, the resonance frequency of each meta-atom is decreased. This is because a higher capacitance value $C_{p}$ is present between consecutive cells in the $y$-direction and also to higher inductance in the circuits. The third parametric sweep concerns the horizontal length $l_{x}$ while keeping $a_{x}$ fixed to $6 \mathrm{~mm}$. An increase in $l_{x}$ causes a decrease in resonance frequency for the different resonators. However, this decrease is more important in the ELC metaatom since vertical arms are present in the structure and therefore a capacitance is created between consecutive cells in the $x$-direction. In the I- and Z-shaped resonators, the decrease is only slight due to the absence of vertical arms. A variation in $l x$ causes inherently a variation in the length of the slant line in the Z-shaped resonator. On the other side, the length of the vertical lines does not vary in the Ishaped structure. Therefore a greater change in inductance is noted with the Z-type resonator and this explains why the Z-type resonator presents a larger shift in frequency than the I-shaped one when $l_{x}$ varies.

\section{Experimental measurement of the resonators}

Prototypes based on the nominal dimensions have been fabricated for each resonator using standard lithography techniques. Total sample size is $18 \times 18$ cells on a $120 \mathrm{~mm} \times$ $120 \mathrm{~mm}$ dielectric board. Microwave measurements based 
on the experimental setup described in ref. 28 have been done on the fabricated prototypes in an anechoic chamber using an Agilent 8722ES network analyzer and two dualpolarized 2-18 GHz wideband band horn antennas. In this case, the two horns are polarized similarly, with the wave vector and electric field polarization directed as shown in Fig. 1. Phase referencing and normalization have been performed in transmission by removing the sample from the signal path, and in reflection, by replacing the sample with a copper plate. The reflection and transmission coefficients obtained from the equivalent circuits of the different resonators are reported in Figs. 3(a) and 3(b). Similar responses are obtained from full-wave simulations performed on the different unit cells as shown in Figs. 3(c) and 3(d). Measured reflection and transmission coefficients are presented in Figs. 3(e) and 3(f).

Calculated and measured magnitudes of $S_{21}$ show clearly a resonance dip for each type of resonator. There is a very good qualitative agreement between simulations and measurements. Table I summarizes the numerical and experimental resonance frequencies.
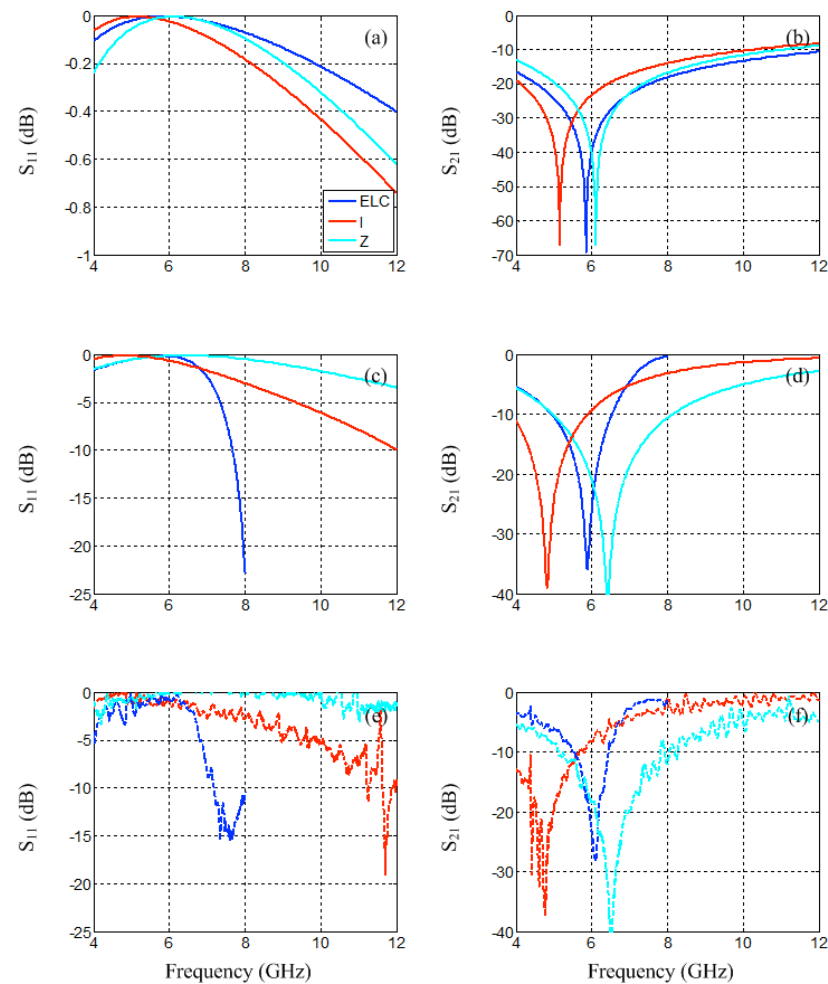

Figure 3: (a)-(b) Calculated from equivalent circuit model, (c)-(d) computed from full-wave simulations, and (e)-(f) measured reflection and transmission responses of the different resonators.

\section{Study of magneto-electric coupling in the Z- shaped resonator}

It can be clearly observed that the ELC and I-shaped resonators possess a high level of symmetry, implying both an electric coupling to an electric field and a magnetic coupling to a magnetic field. However due to the symmetric configuration, the two inductive loops are equivalent but opposite, cancelling the magnetic coupling in the structure
[19]. Thus, a purely electric response is obtained from such structures. In the case of the Z-shaped resonator, the only apparent symmetry is a rotation of $180^{\circ}$ around the $\mathrm{Z}$-axis. Because of the lack of mirror symmetry plane in the Z-type design, the eigenwave in such structures is not as simple as the $y$-direction linear polarized wave defined in Fig. 1. For this reason, cross-polarizing behaviour and magnetoelectric coupling study need to be addressed.

We first start by verifying the electromagnetic (EM) wave polarization of the Z-shaped structure. Two linear eigenmodes corresponding to $x$-polarized and $y$-polarized waves are considered in the numerical simulations. Therefore, four linear transmission coefficients $T_{x x}, T_{y x}, T_{x y}$ and $T_{y y}$ are obtained and used to calculate circular transmission coefficients $T_{++}, T_{-+}, T_{+_{-}}$and $T_{--}$using the following equation [29]:

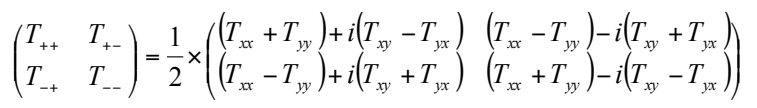
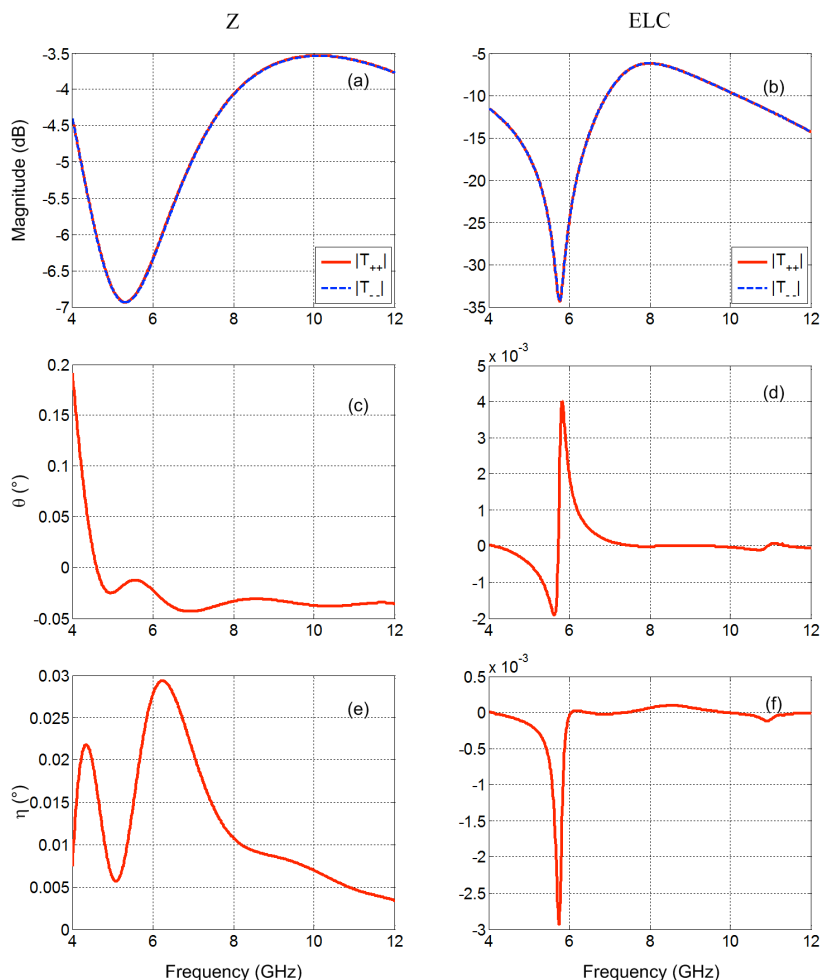

Figure 4: (a)-(b) Computed magnitude of transmission coefficient for the circularly polarized EM wave, (c)-(d) Computed polarization azimuth rotation $(\theta)$, (e)-(f) Computed ellipticity angle $(\eta)$ for the Z-shaped and ELC resonators respectively.

Fig. 4(a) shows the calculated $T_{++}$and $T_{--}$coefficients for the Z-shaped meta-atom. We can note that conversely to the chiral metamaterial presented in Ref. 29, the righthanded and left-handed circularly polarized EM waves are similar in this case. The same behavior is observed for the ELC resonator as illustrated in Fig. 4(b). The polarization azimuth rotation $\theta=\left[\arg \left(T_{++}\right)-\arg \left(T_{--}\right)\right] / 2$, and the 
ellipticity $\quad \eta=\frac{1}{2} \arcsin \left(\frac{\left|T_{++}\right|-\left|T_{--}\right|}{\left|T_{++}\right|+\left|T_{--}\right|}\right)$of elliptically polarized light, are presented in Figs. 4(c) and 4(e) and Figs. 4(d) and 4(f) for the Z-shaped and ELC resonator, respectively. Compared to Ref. 29, the polarization rotation and the ellipticity present very low values close to zero for both the Z-shaped and the ELC resonators, suggesting that the structures do not possess chiral properties.

Further investigations about magnetoelectric coupling are performed by comparing the magnetic field vector distribution in the Z-shaped and ELC resonators at resonance. As it can be observed in Fig. 5, a magnetic loop is formed on each side of the central line in both resonators. The magnetic field vectors of the loops point in opposite directions, cancelling the magnetic coupling in the resonators. It can therefore be deduced that similarly to the ELC resonator, a purely electric response can be obtained from the Z-shaped meta-atom.
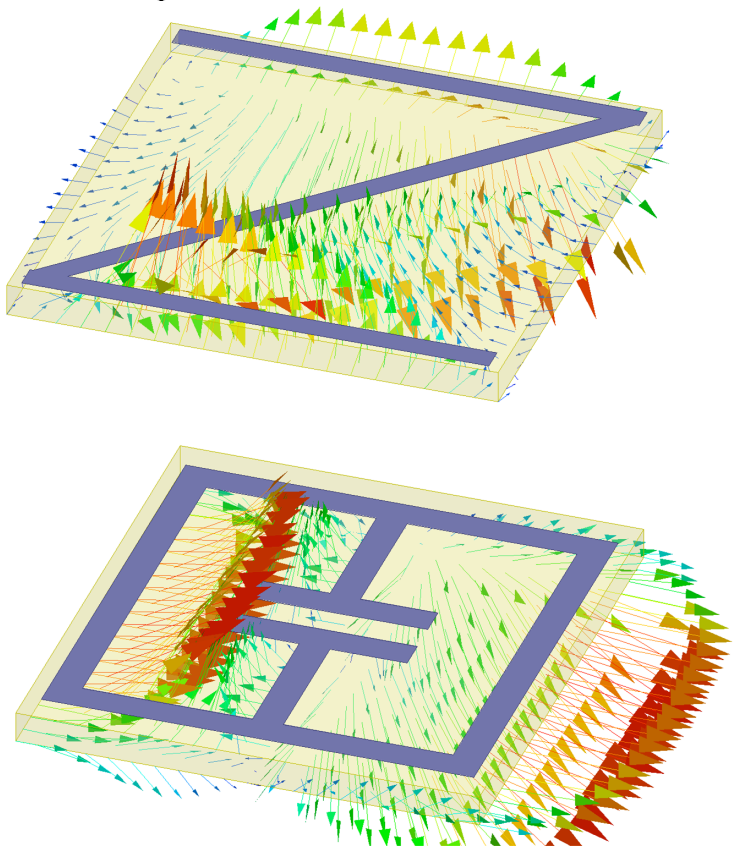

Figure 5: .Magnetic field vector distribution at resonance in the Z-shaped and ELC resonators.

\section{Experimental measurement of bianisotropy}

To experimentally verify bianisotropy effect in the Zshaped meta-atom, cross-polarization transmission measurements are performed on the $18 \times 18$ cells fabricated sample as detailed in [30]. Conversely to co-polarization (or desired polarization) measurements performed in section 4 , here the two horns are polarized orthogonally with respect to each other. The horn antenna used as emitter is polarized with an electric field along the y-direction whereas the one used as receiver is polarized with an electric field along the $\mathrm{x}$-direction, such that the E-plane of one horn is oriented $90^{\circ}$ with respect to the other.

Measurements on the various element samples are presented in Fig. 6(a)-(c) for normal incidence $\left(0^{\circ}\right)$ and in Fig. 6(d)-(f) for $45^{\circ}$ incidence in E-plane. For normalization, co- and cross-polarization measurements are performed with no sample present. It can clearly be observed that in both incidence cases, cross-polarization level of the ELC sample is commensurate with that of no sample present. For the Z-shaped metamaterial sample, cross-polarization is considered negligible below $6 \mathrm{GHz}$ since the level is almost the same as in free space (without the sample). However, the cross-polarization level tends to increase above $6 \mathrm{GHz}$, indicating the presence of magnetoelectric coupling. The resonance frequency of the Z-shaped metamaterial sample having been experimentally measured at $4.9 \mathrm{GHz}$, we can therefore deduce that the latter resonance is purely electric similar to the ELC meta-atom, although the lack of symmetry in the design. Crosspolarization measurements have also been performed on the highly symmetric I-shaped metamaterial and results consistent with the ELC have been observed. $0^{\circ}$

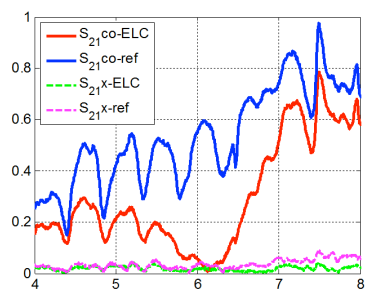

(a)

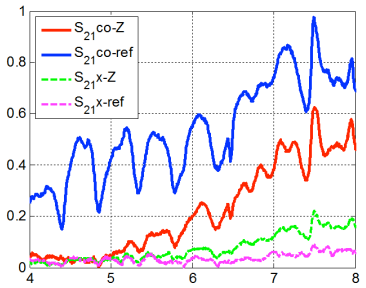

(b)

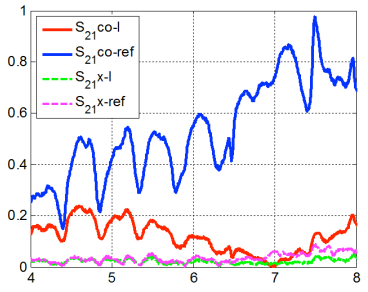

(c) $45^{\circ}$

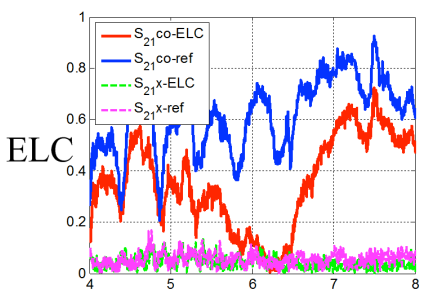

(d)

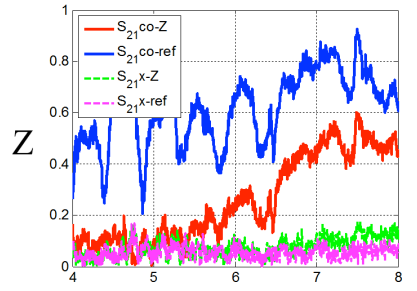

(e)

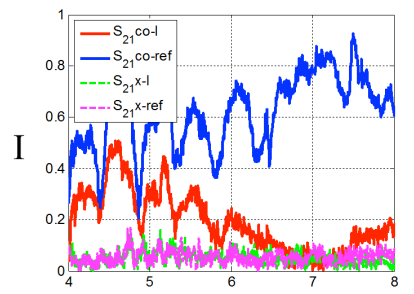

(f)
Figure 6: Comparison of cross-polarization measurements for the various ELC, Z-shaped and I-shaped designs. (a)-(c) $0^{\circ}$ incidence. (d)-(f) $45^{\circ}$ oblique incidence. For calibration, horns aligned and cross-polarization transmission measurements are shown for no sample present (blue and magenta colors).

\section{Effective parameters retrieval}

Using reflection and transmission responses from a single layer of the sample and considering a $y$-direction linear polarized wave, effective parameters can be extracted using the retrieval procedure described in [31]. This is possible since the structure period along the propagation direction is very small compared to the working wavelength. In 
performing the retrieval, we assume a z-direction size $a_{z}=6$ $\mathrm{mm}$ corresponding to a cubic unit cell as proposed in Ref. 19. Extracted permittivity $\varepsilon$ and permeability $\mu$ are shown for ELC, I-shaped, and Z-shaped resonators in the various parts of Fig. 7. Real and imaginary parts of $\mu$ are respectively shown in Fig. 7(a) and 7(b). As stated above, the ELC resonator presents an electric response but no magnetic response, i.e. the real part of $\mu$ is close to unity over the frequencies of interest. However, the retrieved parameters do not strictly comply with this principle. As shown in Fig. 7(a), the real part of $\mu$ becomes anti-resonant. In fact, this anomaly is an artifact introduced during parameter extraction in a frequency range where the medium is highly spatially dispersive [19]. The two other resonators present similar responses. From Figs. 7(c)-7(f), we see a strong electric resonance, with the real part of the permittivity ranging from positive to negative values in the vicinity of the resonance. The values issued from measurement data agree very well with those calculated from numerical simulations. It must be noted that the extraction from the measured spectra are presented without any fitting. From the simulated and measured real parts of $\varepsilon$, it can also be noted that both Z-shaped meta-atoms (with and without gap) present a wider frequency band where the values are negative compared to the ELC resonator. Finally, we stress on the fact that the parameters extracted from the retrieval procedure described in [31] concerning the Zshaped resonator are valid only in the vicinity of the resonance, i.e. for frequencies below $6 \mathrm{GHz}$, since presence of magneto-electric coupling has been observed for higher frequencies.
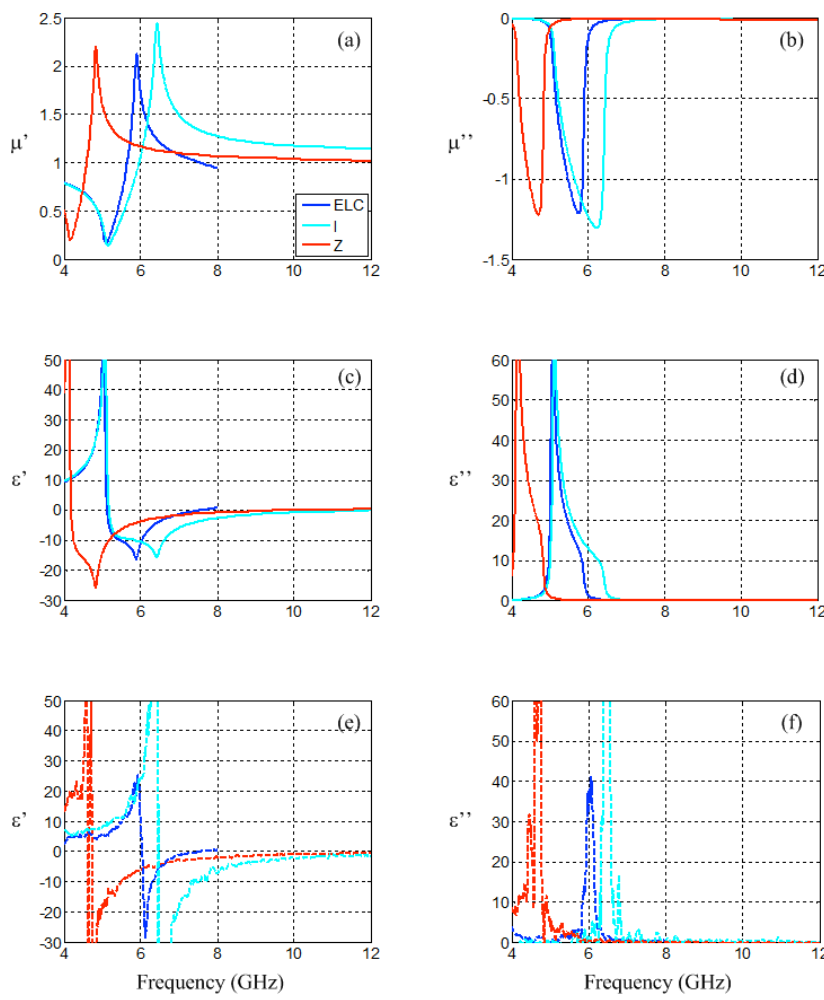

Figure 7: Computed effective parameters: (a) $\operatorname{Re}(\mu)$, (b) $\operatorname{Im}(\mu),(\mathrm{c}) \operatorname{Re}(\varepsilon)$, and (d) $\operatorname{Im}(\varepsilon)$. Measured effective parameters: (e) $\operatorname{Re}(\varepsilon)$ and (f) $\operatorname{Im}(\varepsilon)$.

\section{Conclusions}

We have presented an experimental demonstration of a negative permittivity from various meta-atoms in the microwave domain. The proposed structures, evolved from the transformation of conventional electric-LC (ELC) resonator, are simple to design and can be easily fabricated with standard techniques. A parametric study on the different parameters has also been presented so as to show the influence on the resonance frequency of the different resonators. In particular, a Z-shaped meta-atom has been shown to be an interesting alternative to the conventional ELC. This proposed electric resonator allows to considerably improve the effective medium ratio $\lambda / a$. Due to the simplicity of the structure geometry and to the absence of capacitive gap, a transposition of geometrical parameters can be considered for the optical domain.

\section{Acknowledgements}

This work was supported by the EADS Company Foundation through the METAQOPT project, contract No. 090-AO09-1006. One of the authors (A.D.) acknowledges support for his $\mathrm{PhD}$ scholarship from EADS Company Foundation. The authors thank T. Lepetit and A. Degiron for fruitful discussions on magneto-electric coupling.

\section{References}

[1] U. Leonhardt, Optical conformal mapping, Science 312(5781): 1777-1780, 2006.

[2] J. B. Pendry, D. Schurig, D. R. Smith, Controlling electromagnetic fields, Science 312(5781): 1780-1782, 2006.

[3] D. Schurig, J. J. Mock, B. J. Justice, S. A. Cummer, J. B. Pendry, A. F. Starr, D. R. Smith, Metamaterial electromagnetic cloak at microwave frequencies, Science 314(5801): 977-980, 2006.

[4] W. Cai, U. K. Chettiar, A. V. Kildishev, V. M. Shalaev, Optical cloaking with metamaterials, Nat. Photon. 1(4): 224-227, 2007.

[5] B. Kanté, A. de Lustrac, J.-M. Lourtioz, S. Burokur, Infrared cloaking based on the electric response of split ring resonators, Opt. Express 16(12): 9191-9198, 2008.

[6] H. Chen, B. Hou, S. Chen, X. Ao, W. Wen, C. T. Chan, Design and experimental realization of a broadband transformation media field rotator at microwave frequencies, Phys. Rev. Lett. 102(18): 183903, 2009.

[7] Y. G. Ma, C. K. Ong, T. Tyc, U. Leonhardt, An omnidirectional retroreflector based on the transmutation of dielectric singularities, Nat. Mater. 8(8): 639-642, 2009.

[8] N. Kundtz, D. R. Smith, Extreme-angle broadband metamaterial lens, Nat. Mater. 9(2): 129-132, 2010.

[9] P.-H. Tichit, S. N. Burokur, A. de Lustrac, Waveguide taper engineering using coordinate transformation technology, Opt. Express 18(2): 767-772, 2010. 
[10] S. N. Burokur, M. Latrach, S. Toutain, Theoretical investigation of a circular patch antenna in the presence of a left-handed medium, IEEE Antennas Wireless Propag. Lett. 4: 183-186, 2005.

[11]P.-H. Tichit, S. N. Burokur, A. de Lustrac, Ultradirective antenna via transformation optics, $J$. Appl. Phys. 105(10): 104912, 2009.

[12]P.-H. Tichit, S. N. Burokur, D. Germain, A. de Lustrac, Design and experimental demonstration of a highdirective emission with transformation optics, Phys. Rev. B 83(15): 155108, 2011.

[13] P.-H. Tichit, S. N. Burokur, D. Germain, A. de Lustrac, Coordinate transformation based ultra-directive emission, Elec. Lett. 47(10): 580-582, 2011.

[14] C. Garcia-Meca, A. Martinez, U. Leonhardt, Engineering antenna radiation patterns via quasiconformal mappings, Opt. Express 19(24): 2374323750, 2011.

[15]E. Lier, D. H. Werner, C. P. Scarborough, Q. Wu, J. A. Bossard, An octave-bandwidth negligible-loss radiofrequency metamaterial, Nat. Mater. 10(3): 216222, 2011.

[16]Z. H. Jiang, M. D. Gregory, D. H. Werner, Experimental demonstration of a broadband transformation optics lens for highly directive multibeam emission, Phys. Rev. B 84(16): 165111, 2011.

[17]P.-H. Tichit, S. N. Burokur, A. de Lustrac, Transformation media producing quasi-perfect isotropic emission, Opt. Express 19(21): 20551-20556, 2011.

[18] J. B. Pendry, A. J. Holden, D. J. Robbins, W. J. Stewart, Magnetism from conductors and enhanced non-linear phenomena, IEEE Trans. Microwave Theory Tech. 47(11): 2075-2084, 1999.

[19]D. Schurig, J. J. Mock, D. R. Smith, Electric-fieldcoupled resonators for negative permittivity metamaterials, Appl. Phys. Lett. 88(4): 041109, 2006.

[20] H. Chen, L. Ran, J. Huangfu, X. Zhang, K. Chen, T. M. Grzegorczyk, J. A. Kong, Left-handed materials composed of only S-shaped resonators, Phys. Rev. E 70(5): 057605, 2004.

[21] D. Wang, L. Ran, H. Chen, M. Mu, J. A. Kong, B.-I. $\mathrm{Wu}$, Experimental validation of negative refraction of metamaterial composed of single side paired S-ring resonators, Appl. Phys. Lett. 90(25): 254103, 2007.

[22]A. Sellier, S. N. Burokur, B. Kanté, A. de Lustrac, Negative refractive index metamaterials using only metallic cut wires, Opt. Express 17(8): 6301-6310, 2009.

[23] S. N. Burokur, A. Sellier, B. Kanté, A. de Lustrac, Symmetry breaking in metallic cut wire pairs metamaterials for negative refractive index, Appl. Phys. Lett. 94(20): 201111, 2009.

[24] S. N. Burokur, T. Lepetit, A. de Lustrac, Incidence dependence of negative index in asymmetric cut wire pairs metamaterials, Appl. Phys. Lett. 95(19): 191114, 2009.

[25] T. Koschny, P. Markos, E. N. Economou, D. R. Smith, D. C. Vier, C. M. Soukoulis, Phys. Rev. B 71(24): 245105, 2005.

[26] W. Withayachumnankul, C. Fumeaux, D. Abbott, Compact electric-LC resonators for metamaterials, Opt. Express 18(25): 25912-25921, 2010.

[27]C. Caloz, A. Lai, T. Itoh, The challenge of homogenization in metamaterials, New J. Phys. 7, art. no. 167,2005 .

[28] J. Zhou, L. Zhang, G. Tuttle, T. Koschny, C. M. Soukoulis, Negative index materials using simple short wire pairs, Phys. Rev. B 73(4), 041101(R), 2006.

[29] J. Zhou, J. Dong, B. Wang, T. Koschny, M. Kafesaki, C. M. Soukoulis, Negative refractive index due to chirality, Phys. Rev. B 79(12): 121104(R), 2009.

[30] D. R. Smith, J. Gollub, J. J. Mock, W. J. Padilla, D. Schurig, Calculation and measurement of bianisotropy in a split ring resonator metamaterial, J. Appl. Phys. 100(2): 024507, 2006.

[31]D. R. Smith, S. Schultz, P. Markos, C. M. Soukoulis, Determination of effective permittivity and permeability of metamaterials from reflection and transmission coefficients, Phys. Rev. B 65(19): 195104, 2002. 